\title{
Involucración de personas con discapacidad en proyectos tecnológicos de I+D+i: el caso de APSIS4all
}

\author{
Involvement of people with disabilities in \\ technological R\&D\&i: the case of APSIS4all
}

\section{Resumen}

Este artículo presenta una descripción práctica de las cuestiones y procedimientos necesarios para la involucración de personas con discapacidad en proyectos tecnológicos de $\mathrm{I}+\mathrm{D}+\mathrm{i}$. Se parte de la justificación de esta necesidad, fundamentada en nuevos paradigmas como la investigación inclusiva o emancipatoria. Se realiza un repaso de aspectos prácticos que afectan a las fases y actividades en las que la participación de personas con discapacidad es pertinente (ideación y planificación, recogida de requisitos, diseño y desarrollo y experiencia piloto y validación). Asimismo, se utilizan datos y resultados de un proyecto concreto de I+D+i (APSIS4all) para ilustrar los procedimientos a realizar en cada una de las fases. En conjunto, el artículo sugiere que la participación de personas con discapacidad en un proyecto de estas características no es solo posible sino también deseable.

\section{Palabras clave}

I+D+i, discapacidad, accesibilidad, investigación inclusiva, APSIS4all.

\begin{abstract}
This paper presents a practical description of the issues and procedures required for the involvement of disabled people in R\&D\&i technological projects. It justifies this need, based on new paradigms as inclusive or emancipatory research. A review of practical issues that affect the phases and activities in which the participation of people with disabilities is relevant (ideation and planning, requirements gathering, design and development and pilot and validation) is performed. Also, data and results of a particular R\&D\&i project (APSIS 4 all) are used to illustrate the procedures to be performed in each of the named phases. Overall, the article suggests that the participation of people with disabilities in a project like this is not only possible but also desirable.
\end{abstract}

\section{Keywords}

R\&D\&i, disability, accessibility, inclusive research, APSIS 4 all.
Fausto J. Sainz de Salces <fsainz@gmail.com>

FS UX Consulting

\section{Mercedes Turrero Martín}

$<$ mturrero@consultoria.ilunion. com>

ILUNION Accesibilidad, Estudios y Proyectos.

\section{Lourdes González Perea <lgperea@consultoria.ilunion. com>}

ILUNION Accesibilidad, Estudios y Proyectos

\section{R. Ignacio Madrid}

$<$ nmadrid@consultoria.ilunion. com>

ILUNION Accesibilidad, Estudios y Proyectos

Para citar:

Sainz, F. et al. (2014): "Involucración de personas con discapacidad en proyectos tecnológicos de $\mathrm{I}+\mathrm{D}+\mathrm{i}$ : el caso de APSIS 4 all”, Revista Española de Discapacidad, 2 (2): I2I-I44.

$<$ http://dx.doi.org/I0.5569/23405 I04.02.02.07>

Fecha de recepción: 27-02-20I4 Fecha de aceptación: 29-09-20I4 


\section{Introducción}

\subsection{Las personas con discapacidad y la tecnología}

Las personas con discapacidad encuentran frecuentemente diferentes barreras para el desempeño en su vida diaria, y más en concreto para su participación social. En España, los resultados de la Encuesta de Integración Social y Salud (EISS) (INE, 20I2) muestran que el 9I \% de las personas con discapacidad encuentra problemas para participar en actividades de ocio y culturales, cerca del $58 \%$ para acceder a un empleo adecuado y en torno al $62 \%$ para acceder y moverse por los edificios. En relación con este último problema, el de la accesibilidad al entorno físico, existen abundantes normativas y regulaciones que persiguen que todas las personas puedan llegar a todos los espacios sin sobreesfuerzos y con autonomía (De Benito et al., 2010).

Pero la EISS también muestra que un $28 \%$ de las personas con discapacidad encuentra alguna barrera en el uso de Internet. En este contexto, los avances tecnológicos y de la sociedad de la información se citan frecuentemente como una gran oportunidad para las personas con discapacidad y mayores (en el sentido de oportunidades de participación en la sociedad, acceso a la información y mejora de la calidad de vida en general), aunque también como un riesgo (posibilidad de que una parte importante de la población pueda quedar excluida de estos avances por falta de acceso, capacidad y/o motivación).

A día de hoy existe abundante legislación y normativa técnica sobre accesibilidad tecnológica, lo que permite un aumento paulatino de los niveles de acceso a la web y otras tecnologías entre personas con discapacidad (FVE, 20I3; Technosite, 20II). Sin embargo, el desarrollo tecnológico parece ir más rápido que la eliminación de barreras, y es frecuente que tras la introducción de cada nueva tecnología pasen algunos años sin que sus beneficios lleguen totalmente a las personas con discapacidad. Sirva como ejemplo el desarrollo de la Televisión Digital en España: tras el apagón analógico en el periodo 20092010 no fue hasta el año 2012 que los primeros decodificadores DTV accesibles llegaron a los hogares de las personas con discapacidad. El problema está lejos de quedar solucionado, ya que la nueva generación de televisión digital interactiva y SmartTV nace con importantes barreras de accesibilidad (García Crespo, 20I 2; Jiménez Lara, 20I I).

Es cierto que los nuevos desarrollos legislativos pueden paliar este desfase en un futuro. Por ejemplo, la Convención sobre los Derechos de las Personas con Discapacidad de la ONU cuyo texto fue aprobado en 2006 y ratificado por España en 2008, señala en su artículo 9 que los países deberán "promover el diseño, el desarrollo, la producción y la distribución de sistemas y tecnologías de la información y las comunicaciones accesibles en una etapa temprana, a fin de que estos sistemas y tecnologías sean accesibles al menor costo". Esto tiene su reflejo en el Real Decreto Legislativo I/2013, de 29 de noviembre, por el que se aprueba el Texto Refundido de la Ley General de derechos de las personas con discapacidad $y$ de su inclusión social, donde se explicita que las administraciones públicas deberán "facilitar y apoyar la innovación y el desarrollo de normativa técnica, así como la revisión de la existente, de forma que asegure la no discriminación en procesos, diseños y desarrollos de tecnologías, productos, servicios y bienes, en colaboración con las entidades y organizaciones de normalización y certificación y todos los agentes implicados".

Por todo lo anterior, es necesario que la accesibilidad esté presente no solo a partir del momento de comercialización de las nuevas tecnologías, sino también en las fases previas de investigación y desarrollo. Para ello no basta con el sentido común, sino que es necesaria la aplicación de metodologías que permitan la participación de las personas con discapacidad

I. Naciones Unidas ENABLE ‘www.un.org/spanish/disabilities/default.asp? id $=497$ ? . 
en el proceso, aportando a los desarrollos tecnológicos la necesaria perspectiva de accesibilidad.

\subsection{Participación de personas con discapacidad en I+D+i}

La inversión en investigación, desarrollo e innovación $(\mathrm{I}+\mathrm{D}+\mathrm{i})$ es un pilar fundamental para el progreso de las naciones, especialmente en aquellos aspectos que inciden directamente sobre el bienestar de los ciudadanos. Ciertamente, la investigación sobre cuestiones derivadas de la discapacidad y el envejecimiento de la población es un reto al que debe dedicarse especial atención en el contexto actual.

Sin embargo, y aunque la participación activa de las personas con discapacidad y las organizaciones que las representan se ha ido incrementando y es cada vez más visible en todas las áreas de la sociedad, su implicación en proyectos de $\mathrm{I}+\mathrm{D}+\mathrm{i}$ ha sido menos evidente, a pesar de que las propias personas con discapacidad reconocen esta necesidad de desempeñar un rol más activo como socios y consultores en los proyectos y no solo como meros sujetos de estudio (Kitchin, 2000). Es en este contexto donde surgen nuevos paradigmas de investigación en discapacidad, como la investigación emancipatoria (Barnes, 2003; Vines et al., 20I3) o la investigación inclusiva (Gonzalez Luna, 20I3; Walmsley \& Johnson, 2003).

Con el objetivo de avanzar en esta dirección, se desarrolló el proyecto Euroade (European Research Agendas for Disability Equality) que exploró cómo fomentar la colaboración entre las organizaciones de personas con discapacidad y las instituciones académicas. Los resultados mostraron que era necesaria una mayor participación activa de las organizaciones en identificar y comunicar las prioridades en investigación desde la perspectiva de las personas con discapacidad. Las necesidades de las personas con discapacidad debían ser incorporadas a los programas y convocatorias de investigación europeos (Priestly, 20I0).
En el presente artículo se desarrollan una serie de recomendaciones y buenas prácticas que se extraen, por una parte, de la literatura relevante sobre investigación tecnológica y discapacidad y, por otra, de la extensa experiencia de Technosite ${ }^{2}$, empresa tecnológica de Fundación ONCE especializada en accesibilidad, en el desarrollo de proyectos de I+D+i. Como ejemplo concreto de esta investigación se expone información sobre APSIS 4 all, un proyecto de I+D+i de ámbito europeo orientado a facilitar la interacción con la tecnología de las personas con discapacidad y personas mayores.

\subsection{Diseño centrado en el usuario, diseño universal y diseño participativo}

Existen tres enfoques que pueden ser de interés para aportar metodologías, técnicas y herramientas en la participación de usuarios con discapacidad dentro de proyectos de $\mathrm{I}+\mathrm{D}+\mathrm{i}$ tecnológicos: el diseño centrado en el usuario, el diseño para todos o diseño universal y el diseño participativo.

El diseño centrado en el usuario es un enfoque surgido de la ingeniería del software que trata, mediante diferentes técnicas, de implicar a los usuarios y considerar sus necesidades dentro del proceso de diseño de interfaces. Al diseño centrado en el usuario se le atribuyen una serie de características, como la implicación de un equipo multidisciplinar, la evaluación de los resultados por parte de usuarios o el diseño a través de ciclos iterativos de mejora continua (Sánchez, 20II). El diseño centrado en el usuario es un paso adelante hacia conseguir soluciones más usables, pero no asegura en sí mismo que estas lo sean para todas las personas.

El diseño para todos o diseño universal puede definirse como aquel diseño que desarrolla productos y construye espacios para ser estéticamente idóneos a la vez que usables por la mayoría de la población, salvando las diferencias y limitaciones que puedan provenir de la edad, capacidades u otros

2. Actualmente ILUNION Consultoría. 
factores personales sin la necesidad de diseños especializados o adaptaciones (Steinfeld y Meisel, 20I2). Para la adecuada puesta en práctica de este enfoque es necesaria la involucración de los futuros usuarios de los productos o servicios a desarrollar.

Por último, en el diseño participativo los usuarios potenciales son invitados a colaborar con los diseñadores, investigadores y desarrolladores de productos y servicios tecnológicos durante el proceso de innovación. Aunque la diferencia con los dos enfoques anteriores es a menudo difícil de percibir, el rol de los usuarios en el diseño participativo es más transversal. Así, los usuarios pueden participar en varias fases de un proyecto: durante la exploración inicial y la definición del problema ayudan a definirlo y enfocar las ideas hacia una posible solución, durante el desarrollo ayudan a proponer soluciones, y durante las fases de evaluación validan la adecuación del producto (Muller, 2007; Vines et al., 2013).

A través de estos enfoques de diseño podemos afirmar que se puede empoderar a las personas, y especialmente a las personas con discapacidad, para que sus opiniones sean representadas y escuchadas en los proyectos de $\mathrm{I}+\mathrm{D}+\mathrm{i}$, de forma que los productos y servicios resultantes sean accesibles (Vines et al., 2013).

Existen muchas formas de llevar a cabo actividades de diseño y desarrollo participativo, sobre todo en proyectos de $\mathrm{I}+\mathrm{D}+\mathrm{i}$ : participación en actividades de prueba y validación, consultas, actividades de colaboración y co-diseño, asesoramiento y grupos de enfoque.

Esta es la orientación adoptada en el caso de diferentes proyectos desarrollados por Technosite, quien tiene el compromiso de que todos los participantes colaboren igualmente en la investigación, en un intento de avanzar en esta distribución de responsabilidad: las personas con discapacidad actúan como sujetos en pruebas de usabilidad y accesibilidad, pero también ofrecen su punto de vista sobre sus necesidades y preferencias a través de encuestas, cuestionarios y grupos de enfoque. Además, las oficinas de Technosite en Madrid son un Centro Especial de Empleo, por lo que una parte de los investigadores, diseñadores y desarrolladores que participan en los proyectos de $\mathrm{I}+\mathrm{D}+\mathrm{i}$ son también personas con discapacidad.

En los siguientes apartados se describen diferentes aspectos prácticos a la hora de involucrar a usuarios con discapacidad en proyectos de $\mathrm{I}+\mathrm{D}+\mathrm{i}$, en relación con el reclutamiento, la ejecución de actividades participativas y la comunicación y difusión de resultados.

\section{Antecedentes: ideación y planificación}

Aunque muy frecuentemente tiende a pensarse que la participación de personas con discapacidad se produce entre los límites de inicio y fin de un proyecto, esta debe comenzar mucho antes. Como se ha comentado anteriormente, las ideas para nuevos proyectos deben partir de las necesidades reales de las propias personas con discapacidad y ser canalizadas de la forma adecuada para que puedan cristalizarse en un proyecto de investigación.

Sirva como referencia los siete principios que expone el proyecto FORTUNE y que la Unión Europea (UE) adopta en los programas de investigación del área de e-inclusión (Bühler, 200I):

I. Consorcio: La participación de organizaciones de personas con discapacidad debe ser equitativa respecto al resto de participantes.

2. Basado en organizaciones de usuarios: Los usuarios pertenecen o son representantes de las organizaciones de usuarios. Debe contarse con el apoyo de las organizaciones para establecer contacto con sus miembros.

3. Pago equitativo: La participación de los usuarios debe ser voluntaria, pero también recompensada. La organización 
representante de personas con discapacidad que participa en el proyecto recibe financiación como el resto de socios, y los usuarios individuales reciben compensación por su tiempo y esfuerzo.

4. Accesibilidad: Los materiales, comunicaciones e instalaciones deben ser accesibles para todos los usuarios.

5. Equipo cualificado: Los socios de un proyecto deben proveer equipos con experiencia y una actitud correcta hacia la discapacidad.

6. Planificación adecuada: El plan de proyecto contempla paquetes de trabajo y tareas dedicados a la participación de usuarios.

7. Involucración temprana: Los usuarios son socios del proyecto desde el principio: identificando la idea de proyecto, creando el consorcio y realizando la planificación.

Por tanto, cualquier proyecto de $\mathrm{I}+\mathrm{D}+\mathrm{i}$ debe comenzar desde una adecuada identificación de necesidades y una planificación cuidada de las tareas de involucración de usuarios a realizar para cumplir los objetivos del proyecto.

\section{Reclutamiento de participantes}

En este apartado se analizan cuestiones relacionadas con la selección de participantes y su representatividad, la necesidad de incluir participantes (voluntarios) con discapacidad en los proyectos de $\mathrm{I}+\mathrm{D}+\mathrm{i}$, el acercamiento a este grupo y el contacto con los potenciales participantes $\mathrm{y}$, finalmente, algunas consideraciones éticas y legales a tener en cuenta.

\subsection{Representatividad de la muestra y cuotas de participación}

El problema de la representatividad en los estudios de discapacidad no es solo propio de los proyectos de $\mathrm{I}+\mathrm{D}+\mathrm{i}$ tecnológica, sino que afecta también a la propia investigación social (Alaminos y Castejón, 2006). A menudo, la persona con discapacidad es definida como poseedora de unas características funcionales estándar que rara vez representan el amplio abanico de perfiles existentes. Este enfoque se muestra poco eficaz desde la perspectiva del diseño para todos, cuyo objetivo es dar respuesta al mayor rango posible de necesidades existentes. En este sentido, Toboso-Martín y Rogero-García (2OI2) señalan como principales retos para incorporar la filosofía del diseño para todos a la investigación social el aumento de la calidad de los diseños metodológicos y la eliminación de barreras que pueden impedir la participación de determinados perfiles en las investigaciones.

En el diseño muestral de los proyectos de I+D+i llevados a cabo en Technosite, la técnica con mayor aplicabilidad es el muestreo estratificado. Es necesario señalar que aunque no hay un criterio único a la hora de establecer subpoblaciones objetivo o variables definitorias de la muestra de personas con discapacidad, el diseño muestral parte de un conocimiento profundo de las características y variabilidad de cada grupo. Por otra parte, los requisitos de representatividad de la muestra y las cuotas de participación concretas dependen en gran medida del alcance, objetivos y destinatarios de cada proyecto. A continuación se señalan dos ejemplos:

- $\quad$ En el proyecto INREDIS 3 , con un alcance muy amplio en cuanto al análisis de entornos, contextos de uso y tecnologías, se trató de ofrecer una clasificación de personas con discapacidad y mayores, a partir de una serie de categorías homogéneas y operativas a la hora de comprender las dificultades de accesibilidad de cada colectivo. Así, las cuotas se establecieron para cada uno de los siguientes grupos: discapacidad visual, discapacidad auditiva, discapacidad para el desplazamiento, discapacidad para la manipulación, discapacidad intelectual y autismo, enfermedad mental, mayores y otras condiciones especiales.

3. INREDIS Interfaces de relación entre el entorno y las personas con discapacidad «www.inredis.es〉. 
- Con un alcance más limitado, el proyecto $\mathrm{SAAPHO}^{4}$ analizó los requisitos de la población mayor en el uso de sistemas de teleasistencia y domótica. Al estar dirigido a una población tan acotada, las principales variables para establecer cuotas de participación fueron la edad, género y experiencia previa con la tecnología (Domenech et al., 20I3).

Es necesario asegurarse de que la muestra de la población contiene representación de todos los perfiles. Para ello es muy probable que se tenga que hacer frente a varios obstáculos al tratar de obtener la colaboración de determinados grupos, como se describe a continuación.

\subsection{Información del proyecto y acercamiento a participantes}

Aunque pueda parecer que los usuarios con discapacidad estén dispuestos a participar en cualquier investigación que les beneficie directamente, las cosas no son tan sencillas, según nuestra experiencia en diferentes proyectos. La falta de tiempo, la disponibilidad y la motivación de los participantes así como los aspectos logísticos deben ser cuidadosamente consideradas para valorar las posibilidades de que una persona participe en las actividades de investigación. Una aparente falta de propósito puede ser un elemento disuasorio para los posibles voluntarios a la hora de participar en los proyectos de $\mathrm{I}+\mathrm{D}+\mathrm{i}$, por lo que la presentación a los usuarios de los posibles beneficios derivados de su colaboración puede mejorar en gran medida las posibilidades de establecimiento de un compromiso por su parte.

Por tanto, una vez definida la muestra, un paso más en el diseño del reclutamiento consiste en la elaboración de la documentación sobre el proyecto $\mathrm{I}+\mathrm{D}+\mathrm{i}$ que se quiere dar a conocer a los potenciales participantes. Esta debe incluir la información más relevante sobre los objetivos y el desarrollo del proyecto en cuestión, así

4. SAAPHO Secure Active Aging: Participation and Health for the Old ‘www.saapho-aal.eus. como los datos de contacto del responsable del reclutamiento para que las personas interesadas puedan ampliar la información. Lo habitual en la práctica de Technosite es incluir los siguientes datos:

- La descripción y objetivos del proyecto, así como los resultados esperados e implicaciones para las personas con discapacidad.

- Los requisitos de los participantes (sus características socio-demográficas).

- Las tareas de los participantes (qué tareas deberán realizar para colaborar en el proyecto, tales como participar en grupos de discusión, rellenar cuestionarios, realizar entrevistas o participar en pruebas de tecnología).

- Las compensaciones y costes (declarar los costes asociados o la compensación económica o en especie que reciben los participantes, o su ausencia en el caso de que no se contemplen).

- Los beneficios potenciales, riesgos o molestias asociadas a la participación.

- El destino de los datos y resultados del proyecto.

Con la información del proyecto ya definida, comienza la fase de acercamiento o identificación de los participantes concretos. En este punto, la persona responsable del reclutamiento recurre a los diferentes contextos en los que puede acceder a los perfiles establecidos en la muestra y difunde la información del proyecto para conseguir su colaboración. Se exploran tres tipos de contextos:

- Físico: Cuando el responsable del reclutamiento hace acto de presencia en el lugar o lugares en donde viven, trabajan o desarrollan cualquier tipo de función significativa los miembros de los colectivos que forman parte del estudio. Por ejemplo, las organizaciones de personas con discapacidad o los centros especiales de empleo.

- Social: Acercamiento y establecimiento de algún tipo de relación con las 
organizaciones de las que forman parte los individuos seleccionados, que en este caso realizan funciones de actores intermediarios en el reclutamiento. La comunicación con estos intermediarios puede hacerse personalmente o a través de llamadas telefónicas o correos electrónicos. En este contexto, el responsable del reclutamiento puede ayudarse de sus "redes personales" o de la "activación de redes sociales" que pueda llevar a cabo todo el equipo de investigación. Esto es, hacer recuento de conocidos que pudieran responder a estos perfiles o que, al menos, pudieran dar información para localizar a estos participantes.

- Virtual: Acercamiento a los colectivos a través de la presencia en páginas web, redes sociales, foros o chats en los que puedan participar individuos con el perfil solicitado. Para este contexto, en nuestra práctica habitual se elaboran noticias y banners que se publican en el portal Discapnet ${ }^{5}$, en la página web del proyecto en cuestión (si cuenta con ella), y/o en los portales web de las organizaciones de personas con discapacidad de los perfiles requeridos.

Aunque es deseable que las propias organizaciones de personas con discapacidad participen como socios de los proyectos de $\mathrm{I}+\mathrm{D}+\mathrm{i}$, hay ocasiones en las que no es así o bien las organizaciones que participan no cubren todos los perfiles que es necesario involucrar. En esos casos se suele acudir a las organizaciones de usuarios en el papel de mediadoras entre el proyecto y las personas con discapacidad, de forma que puedan hacer llegar las informaciones y facilitar el reclutamiento. Aquí debe reconocerse que las organizaciones tienen un papel muy importante en este acercamiento, y en muchos casos pueden facilitar (también limitar) la participación. En cualquier caso, es la persona como individuo quien tiene la última palabra para participar en un proyecto (Stalker, I998).

5. DISCAPNET El portal de las personas con discapacidad ‘www.discapnet.es'.
Una práctica común entre las organizaciones de investigación para la búsqueda de participantes es recurrir a bases de datos existentes (empresas con paneles de usuarios y bases de datos de instituciones). Desde 2007, el área de Estudios e Investigación Social de Technosite cuenta con una base de datos con el registro de personas con discapacidad que han participado en proyectos de investigación y están dispuestas a seguir participando. Sin embargo, el uso de bases de datos tiene también desventajas: algunas personas participan frecuentemente en actividades de investigación, y con el tiempo corren el riesgo de adquirir sesgos de respuesta y/o mostrar desinterés por estas actividades. Es por ello que este factor (el haber participado previamente en actividades de investigación o no) suele considerarse para la conformación de las muestras de forma que siempre haya personas con diferente experiencia de participación en proyectos de $\mathrm{I}+\mathrm{D}+\mathrm{i}$.

\subsection{Contacto, convocatoria y consentimiento informado}

Una vez que el responsable del reclutamiento se ha acercado al "lugar" o al "grupo" de referencia de las "unidades muestrales", llega el momento de contactar directamente con estas personas, es decir, dirigirse personalmente al candidato a participar en la investigación y asegurarse de que cumple las condiciones para formar parte de la muestra y tiene toda la información que necesita.

Como se ha reflejado en el apartado anterior, los candidatos pueden provenir de diferentes fuentes de selección:

I. De la búsqueda activa de participantes, contando incluso con la presencia física del reclutador en el entorno de las personas con discapacidad.

2. De la difusión de la información del proyecto a través de portales web y otros medios.

3. De una selección de la organización de referencia, que ha actuado como intermediario. 
4. De bases de datos de participantes en actividades de investigación previas.

Independientemente de cómo se haya iniciado el acercamiento, a la hora de dirigirnos personalmente a un candidato/a pueden utilizarse varios canales:

- Contacto cara a cara

- Contacto telefónico

- Contacto por correo electrónico

La accesibilidad de las comunicaciones debe ser tenida en cuenta en este paso. Por ejemplo, en el caso de personas con discapacidad auditiva es preferible ponerse en contacto a través del correo electrónico, programas de mensajería o SMS.

Una vez que se ha establecido el primer contacto, se deben realizar algunas preguntas para confirmar que el candidato en cuestión cumple con los requisitos del perfil que se ha solicitado. Además, en caso de que se hubieran solicitado varios perfiles, se le harán unas preguntas adicionales para confirmar a cuál de los distintos perfiles solicitados responde mejor el individuo.

Esto suele hacerse a través de un cuestionario de captación o reclutamiento, más o menos estructurado o informal, escrito u oral.

Si la persona cumple con los requisitos, llega el momento de lanzar la convocatoria. Aunque la persona ya conocerá información genérica sobre el proyecto y el tipo de actividades que se realizan, es necesario recordar esta información y describir las actividades concretas en las que va a participar. El texto de la convocatoria debe incluir los siguientes puntos:

- Proporcionar información sobre la investigación y la entidad que la lleva a cabo, sin desvelar el objetivo explícito de la misma (ello podría orientar respuestas posteriores).

- Explicar brevemente en qué consiste la participación.

- Indicar la fecha, la hora y el lugar de realización de la reunión, la entrevista, etc.
- Informar de la duración de la reunión.

- Transmitir la importancia y valor de participar.

- Informar de la gratificación y/o compensación de costes.

- Se aconseja incluir indicaciones para llegar al punto de reunión o prueba, como un mapa o transportes cercanos.

- Preguntar nombre y teléfono o e-mail a los convocados para confirmar su asistencia horas antes de la sesión o comunicar imprevistos.

Generalmente, es aconsejable hacer llegar estas condiciones por escrito, por ejemplo, a través del correo electrónico. Debe asegurarse que la documentación electrónica que se envíe (por ejemplo documentos Word o PDF) es accesible, utilizando un lenguaje sencillo y evitando el uso de terminología técnica.

En el caso de las personas con discapacidad, es importante preguntar por necesidades específicas de apoyo. En algunos casos, será necesario apoyar la movilidad abonando el desplazamiento a través, por ejemplo, de un servicio de Eurotaxi o taxi convencional, o acompañando a la persona hasta un punto de encuentro conocido o estación de transporte público de fácil acceso.

Para las personas con discapacidad intelectual, suele ser necesario contactar previamente con el tutor o con un profesional cercano a la persona que ofrezca apoyo sobre su posible participación. En todo caso, los mensajes que transmitamos a estos candidatos han de ser sencillos y, si fuera necesario, en formato de Lectura Fácil.

Para la correcta gestión de participantes en un proyecto, es necesario tener en cuenta determinados aspectos éticos y legales (Sainz de Salces y Bustamante, 20I4). Una cuestión imprescindible es la elaboración y recogida de los consentimientos informados. Estos deberán describir los derechos que asisten a los voluntarios, los objetivos de cada actividad, el papel de los participantes en la misma y el tratamiento que se hará con los datos que 
se recojan. Desde el punto de vista legal y considerando la Ley Orgánica de Protección de Datos, se entiende que a partir de la información que recibe la persona antes de participar en un proyecto, el afectado es consciente y toma conocimiento de la existencia del tratamiento de datos que se realizará. El consentimiento debe ser firmado por el interesado, por su tutor legal o un familiar en caso de que la persona necesite de este apoyo.

\section{Participación en actividades de investigación}

Una vez superadas las barreras de comunicación y acceso de las personas con discapacidad a los proyectos, es igualmente necesario facilitar la participación en determinadas actividades de investigación.

\subsection{Modalidades de participación. Técnicas y herramientas}

Como se ha indicado previamente (Vines et al., 20I3), la aplicación de un enfoque participativo para involucrar a personas con discapacidad en los proyectos de $\mathrm{I}+\mathrm{D}+\mathrm{i}$ puede requerir cierto grado de innovación metodológica y/o cuestionar los diferentes papeles desempeñados por los investigadores y los participantes.

Por otra parte, la intensidad y contenido de la participación depende en cierta medida de la fase del proyecto en la que esta se encuadre. La mayoría de los proyectos de $\mathrm{I}+\mathrm{D}+\mathrm{i}$ se pueden estructurar en las siguientes fases:

I. Fase de recogida de requisitos: El objetivo es frecuentemente 'descubrir' las necesidades y preferencias de uno o varios colectivos de usuarios respecto a la tecnología. Las técnicas más empleadas en esta fase son las encuestas, los focus groups o grupos focales y las entrevistas a usuarios e informantes clave.
2. Fase de diseño y desarrollo: El diseño y desarrollo de productos y servicios tecnológicos desde una perspectiva de diseño centrado en el usuario se realiza a partir de ciclos iterativos, en los que la participación de las personas con discapacidad es imprescindible para detectar errores y barreras y en definitiva para incorporar mejoras en el producto final. En este punto es frecuente la participación en pruebas de usuario de los prototipos que están siendo desarrollados, donde se recogen datos objetivos y subjetivos en una serie de dimensiones de usabilidad y accesibilidad que afectan a la experiencia de usuario (Tullis \& Albert, 2008).

3. Fase de experiencia piloto y validación: Finalmente, es necesario analizar la experiencia de los usuarios en un entorno real (como contraposición a las pruebas realizadas en laboratorio en la fase anterior). Aquí las técnicas de investigación se dirigen a la validación de la solución tecnológica, empleándose técnicas como las encuestas o las entrevistas en profundidad.

\subsection{Comunicación con los participantes y recogida de datos}

Como se ha comentado, una de las principales barreras para la participación de personas con discapacidad en las actividades de investigación se encuentra en la comunicación con determinados perfiles. Es necesario tener en cuenta que el desarrollo de la prueba, entrevista o grupo de discusión requiere ser adaptado para producir la mayor cantidad de información posible atendiendo a las necesidades del interlocutor. El criterio fundamental es comprender la disposición, las limitaciones o diferencias en los funcionamientos de los usuarios que tenemos delante para que la obtención de los datos sea lo más provechosa posible.

Por ejemplo, para el caso de grupos de discusión o co-diseño y en las entrevistas donde participen personas de diferentes perfiles: 
- En la conversación cara a cara, es probable que las personas con discapacidad auditiva nos lean los labios, de manera que debemos evitar taparnos la boca o mirar a otra parte mientras les hablamos. No obstante, no se debe dar por hecho que un participante sordo va a saber leer los labios, sino que en la fase de contacto debemos haberle preguntado cuál es su principal sistema de comunicación y si necesita intérprete de lengua de signos o cualquier otro apoyo a la comunicación. En este último caso, el intérprete deberá también estar presente en la sesión o entrevista.

- En las sesiones donde se muestran imágenes, vídeos o gráficos es necesaria una correcta audiodescripción de los contenidos para personas con discapacidad visual.

- En el caso de las personas con discapacidad cognitiva, la formulación de las preguntas y la conversación (sugerencias y provocaciones) deben adaptarse a su capacidad de abstracción. El uso de fotografías ha demostrado ser muy útil en este caso: permiten situar al participante en el contexto de la investigación y pueden servir para orientar sus respuestas de manera más precisa (Young, 2006).

- En lo relativo a las personas con discapacidades físicas, debe asegurarse que las instalaciones son accesibles. En el caso de grupos de discusión, el mobiliario permitirá a la persona participar en igualdad de condiciones que el resto de asistentes.

En relación con el uso de cuestionarios, muy utilizados en las pruebas de usabilidad y accesibilidad de tecnología, requieren de una adaptación para ser usados por personas con diferentes perfiles:

- En caso de personas con discapacidad cognitiva, se debe simplificar al máximo las preguntas y las categorías de respuesta. Se recomienda apoyar las opciones de respuesta, en la medida de lo posible, con referentes visuales. Esto puede ser especialmente útil cuando hay más de dos categorías. Al entrevistado se le ofrecen, además de las categorías escritas, iconos que aluden a cada una de ellas, de modo que puede ser más fácil identificar la respuesta deseada.

- En el caso de las personas ciegas, el cuestionario debe estar escrito en braille, o bien ofrecerlo en formato electrónico junto a un lector de pantalla que le permita leer los enunciados y opciones de respuesta.

- Para personas con discapacidad auditiva, una buena opción puede ser aplicar el cuestionario a través del correo electrónico o de una web diseñada para ello. Si se opta por la vía del cuestionario heteroadministrado, los administradores de la prueba o entrevistadores deben conocer el lenguaje de signos o contar con la ayuda de un intérprete y haber recibido una formación específica para administrar el cuestionario.

- Para las personas familiarizadas con las tecnologías de la comunicación y que dispongan de hardware y software adaptados, la web o el correo electrónico puede ser un buen mecanismo de recogida de información. Sin embargo, esta vía no es aconsejable si el objetivo es captar información que represente a todo el colectivo estudiado, ya que el porcentaje de personas con discapacidad que no usan Internet con destreza suficiente es más elevado que en el resto de la población.

Respecto a la duración de las sesiones de recogida de datos, en el caso de personas que se fatigan con facilidad, los entrevistadores/ administradores deben ser flexibles y ofrecer descansos o incluso cancelar la entrevista, en caso necesario (Harris y Roberts, 2003). Ese posible cansancio podría provocar a su vez sesgos en la información recogida.

En suma, los investigadores, especialmente aquellos en contacto directo con los participantes 
con discapacidad, tienen que ser flexibles y hábiles para extraer la información adecuando el proceso a las necesidades de estos participantes.

\subsection{Dificultades frecuentes}

El reclutamiento de participantes y su involucración en la investigación consume bastante tiempo y recursos. Lo primero a destacar en este sentido, es que el trabajo con personas con discapacidad requiere tiempo y recursos adicionales a los requeridos con población sin discapacidad, especialmente si se trata de actividades con personas con discapacidad cognitiva (Stalker, I998).

Por otro lado, aunque los beneficios del diseño participativo estén bien establecidos, es probable que durante el desarrollo de proyectos y actividades de investigación participativa surjan conflictos de interés entre las partes interesadas (participantes, voluntarios, patrocinadores, clientes, asesores, etc.) (Safayeni et al., 2008). Estos conflictos se producen no sólo entre los participantes y moderadores, sino también a menudo entre las diferentes partes del conglomerado investigador: entre las organizaciones de usuarios y otros miembros del consorcio del proyecto, entre investigadores y grupos políticos, entre investigadores y gestores de la investigación, y otras combinaciones posibles. También se dan situaciones éticas complejas en la relación entre los investigadores e investigados que deben ser tenidas en cuenta (Aldred, 2008).

Durante el reclutamiento de los participantes con discapacidad nos encontramos con diferentes obstáculos, entre los que pueden citarse:

- La cuota de determinados perfiles específicos puede ser difícil de alcanzar, ya que en algunos casos tienen dificultades evidentes para desplazarse o comunicarse.

- La logística es una preocupación importante, especialmente para personas ciegas, con visión parcial y aquellas con movilidad reducida.
- El consentimiento informado levanta a veces suspicacias o se prevé como un posible problema, especialmente cuando es necesario involucrar a personas con tutor legal o menores de edad.

- En ocasiones, hay dificultad para concertar citas debido al tiempo limitado por ambas partes (investigadores y participantes).

- Personas no familiarizadas con la tecnología son reacias a participar, por miedo a que su competencia sea evaluada.

- Es frecuente la cancelación de las citas, que implican un retraso y la posibilidad de dejar de contar con la participación de esa persona en el proyecto.

En relación con este último punto, señalar que las cancelaciones pueden tener su origen en diferentes agentes del proyecto (la tecnología puede haber fallado y no estar lista para ser mostrada, los investigadores pueden haber sufrido algún contratiempo que retrase el trabajo o las personas participantes pueden tener algún imprevisto). En estos casos es importante comunicar los retrasos con la antelación suficiente, y dar diferentes opciones para poder retomar su participación en el futuro.

\section{Comunicación y difusión de resultados}

Las actividades de difusión son esenciales para garantizar el impacto esperado de los resultados de la investigación. El público objetivo, al que se dirigen las actividades de comunicación y difusión de los proyectos de investigación tecnológica, suele estar compuesto por:

- Los usuarios finales (sociedad en general y el colectivo de personas con discapacidad en particular).

- Los profesionales y las organizaciones de usuarios (por ejemplo, asociaciones de personas con discapacidad).

- Los centros de investigación, universidades y organismos de normalización.

- Organismos públicos. 
- Las organizaciones de la industria y los proveedores de servicios.

- $\quad$ Las organizaciones tecnológicas.

Las actividades de difusión están destinadas a mantener a estos actores comprometidos e informados de los resultados del proyecto en función de la naturaleza e intereses de cada grupo.

Pero frecuentemente, los resultados de los proyectos de $\mathrm{I}+\mathrm{D}+\mathrm{i}$ se difunden únicamente entre expertos, en congresos y/o a través de artículos científicos, y esta información no llega a los destinatarios finales. Los participantes se pueden decepcionar por no ver los resultados del proceso y de su aportación. Por ello, se debe hacer hincapié en que la transferencia de conocimiento llegue a este grupo y a su entorno.

Una razón adicional para mantener informados a los participantes en un proyecto es que es necesario que las personas con discapacidad participen no solo en una actividad concreta, sino durante un periodo más largo, cubriendo la duración de un proyecto. Por ello, es imprescindible informar de los avances y resultados, para lo cual se cuenta con la ayuda de herramientas de difusión como las redes sociales, listas de correo, foros, etc.

El sitio web del proyecto debe ser la principal referencia para consultar la información y resultados. Es imprescindible que el diseño y contenidos se adecuen a todos los públicos y que la web sea accesible para que cualquier persona pueda consultar y entender la información. Para ello, se ha de aplicar el estándar internacional de referencia: Pautas de Accesibilidad para el Contenido en la Web 2.o, definidas por el $\mathrm{W}_{3} \mathrm{C}^{6}$.

Además es importante que los principales resultados y eventos del proyecto se comuniquen a través de noticias para poder difundirlas en portales web o redes sociales relacionadas con la temática del proyecto y de referencia para el público objetivo. Por ejemplo, para los usuarios

6. Web Content Accessibility Guidelines (WCAG) 2.0: ‘www. w3.org/TR/2008/REC-WCAG2O-2008 I 2 I I/. . finales con discapacidad en los proyectos desarrollados desde Technosite se suelen publicar estas noticias en el portal Discapnet, un portal que ofrece contenidos y servicios específicos para las personas con discapacidad, con más de I 2 millones de visitas al año.

6. El proyecto APSIS4all: un caso práctico de involucración de personas con discapacidad en proyectos I+D+i:

\subsection{Descripción del proyecto}

El proyecto $\mathrm{APSIS}_{4}$ all $^{7}$ es un proyecto dirigido a superar barreras de accesibilidad a través de la personalización de terminales de acceso público como los cajeros automáticos y las máquinas expendedoras de títulos de transporte. Se trata de que estos dispositivos se adapten a las necesidades y preferencias de cada usuario, permitiendo nuevas formas de interacción con estos dispositivos a través de tarjetas contactless o teléfonos móviles inteligentes. Este proyecto se ejecuta a nivel europeo y desarrolla dos experiencias piloto a gran escala: una relacionada con las máquinas expendedoras de títulos de transporte en la ciudad de Paderborn (Alemania), y la otra con el uso de cajeros automáticos accesibles, en el que 'la Caixa' ha desplegado más de ıоo० cajeros automáticos en Madrid y Barcelona desde finales de 20I3.

El funcionamiento en el piloto español es el siguiente. El primer lugar, el usuario accede a una aplicación web donde especifica sus necesidades y preferencias respecto al uso de la tecnología ${ }^{8}$. En esta aplicación es posible cambiar el tamaño del texto, la combinación de colores de la pantalla, activar un lector de pantalla, incluir contenido en lenguaje de signos o simplificar la interacción con el terminal,

7. APSIS 4 all Accessible Personalised Services in Public Digital Terminals for all ‘www.apsis4all.eu'.

Proyecto liderado por Technosite y financiado por la Unión Europea a través del programa ICT-PSP como parte del Programa Marco de Competitividad e Innovación. GA 270977 8. ‘http://cajerofacil.apsis4all.eu〉. 
entre otras características. La información se almacena posteriormente en una tarjeta contactless, la cual permite acceder al cajero acercando la tarjeta a un lector y sin necesidad de introducirla en una ranura (lo cual elimina ya algunas barreras de accesibilidad para, por ejemplo, personas con problemas de destreza). Estas opciones de personalización están disponibles para todos los clientes, que pueden elegir el tipo y formato de la información que reciben a través del cajero automático. Sin embargo, APSIS $_{4}$ all será especialmente útil para las personas con algún tipo de discapacidad, aquellas de edad avanzada, las no familiarizadas con la tecnología, las que tengan algún problema transitorio de movilidad o los extranjeros con diferencias culturales importantes.

\subsection{Reclutamiento de participantes}

El punto de partida para establecer la muestra de participantes con discapacidad es delimitar los distintos perfiles que reflejen la realidad y los diversos puntos de vista de los usuarios ante la tecnología o dispositivos que se están investigando. Para este proyecto, se establecieron los perfiles de acuerdo con los resultados de un estudio previo sobre usos, aceptabilidad y preferencias respecto a los cajeros automáticos (Fundación ONCE, 2009).

Los perfiles de participantes considerados para APSIS $_{4}$ all, de acuerdo con este estudio, fueron:

- Personas con ceguera

- Personas con baja visión

- Personas sordas

- Personas con hipoacusia

- Personas con discapacidades físicas

- Personas con discapacidades cognitivas

- Personas sin discapacidad de más de 65 años (personas mayores)

- Personas sin discapacidad de menos de 65 años

Además de los perfiles de discapacidad, se tuvieron en cuenta otras variables para la definición de la muestra, como son el género, la edad y el nivel educativo.
El siguiente paso tras definir los perfiles a los que el proyecto se dirige, es informar sobre el mismo tratando de incidir especialmente en los perfiles objetivo. En muchas ocasiones los proyectos $\mathrm{I}+\mathrm{D}+\mathrm{i}$ generan información donde abundan los términos técnicos, lo cual supone una barrera para la comprensión de muchas personas. Por ello, un primer paso para dar a conocer el proyecto y contactar a posibles usuarios de APSIS4all fue la redacción de una hoja informativa con los principales objetivos y características del proyecto para su distribución.

Esta hoja, que además de presentar el proyecto incluía un enlace para rellenar una encuesta online, se distribuyó a través de diferentes medios:

- Publicación de nota de prensa en el portal Discapnet ${ }^{9}$ y difusión a través de redes sociales (Twitter, Facebook, Blogs, etc.)

- Contacto telefónico o electrónico con organizaciones de personas con discapacidad y mayores en Madrid y Barcelona (por ejemplo FESOCA, COCEMFE, COCARMI, ONCE,

Associació Lectura Fàcil, etc.)

- Por correo electrónico, a través de bases de datos de personas que han participado en proyectos previos del área de Estudios e Investigación Social de Technosite.

El uso de diferentes medios de distribución de la encuestra trata de paliar algunos sesgos que podían afectar a la muestra, como pueden ser la pertenencia o no a diferentes asociaciones, el uso activo de internet y otras tecnologías o la experiencia previa como participante en proyectos de investigación.

Esta primera Fase de recogida de requisitos tuvo como resultado la participación de un total de 225 personas ( 166 en encuestas, 36 entrevistas en profundidad y 23 tests de usuario). La muestra de personas con discapacidad supuso el $80 \%$ del total. En la siguiente tabla se muestra la

9. Un ejemplo de nota de prensa puede verse aquí ‘www.discapnet.es/Castellano/Actualidad/Discapacidad/Paginas/detalle. aspx? Noticia $=273622$ '. 
distribución por perfiles de los participantes en la actividad con mayor número de participantes, una encuesta on-line.

\begin{tabular}{|l|c|}
\hline Perfil & $\begin{array}{c}\mathbf{N}^{\circ} \text { de } \\
\text { participantes }\end{array}$ \\
\hline Ceguera & 18 \\
\hline Baja visión & 29 \\
\hline Personas sordas & 14 \\
\hline Personas con hipoacusia & 11 \\
\hline $\begin{array}{l}\text { Personas con discapacidades } \\
\text { físicas }\end{array}$ & 33 \\
\hline $\begin{array}{l}\text { Personas con discapacidades } \\
\text { cognitivas }\end{array}$ & 16 \\
\hline $\begin{array}{l}\text { Personas sin discapacidad } \\
\text { (mayores de 65 años) }\end{array}$ & 13 \\
\hline $\begin{array}{l}\text { Personas sin discapacidad } \\
\text { (menores de 65 años) }\end{array}$ & 43 \\
\hline Total ${ }^{9}$ & 166 \\
\hline
\end{tabular}

Esta primera encuesta facilitó enormemente el trabajo para actividades de investigación posteriores. En ella participaron un total de I66 personas, la mayoría de las cuales accedieron a seguir colaborando con APSIS4all, siendo posible seleccionar aquellos perfiles necesarios para actividades posteriores en función de su perfil sociodemográfico.

Por ejemplo, para la Fase de diseño y desarrollo de la herramienta de personalización y de las distintas interfaces del cajero automático, se seleccionaron un total de Ioo personas. La siguiente tabla muestra su distribución por perfiles:

\begin{tabular}{|l|c|}
\hline Perfil & $\begin{array}{c}\mathbf{N}^{\circ} \text { de } \\
\text { participantes }\end{array}$ \\
\hline Ceguera & 16 \\
\hline Baja visión & 16 \\
\hline Personas sordas & 15 \\
\hline Personas con hipoacusia & 7 \\
\hline $\begin{array}{l}\text { Personas con discapacidades } \\
\text { físicas }\end{array}$ & 14 \\
\hline $\begin{array}{l}\text { Personas con discapacidades } \\
\text { cognitivas }\end{array}$ & 13 \\
\hline $\begin{array}{l}\text { Personas sin discapacidad } \\
\text { (mayores de 65 años) }\end{array}$ & 10 \\
\hline $\begin{array}{l}\text { Personas sin discapacidad } \\
\text { (menores de 65 años) }\end{array}$ & 100 \\
\hline \begin{tabular}{l} 
Total \\
\hline
\end{tabular} & 9 \\
\hline
\end{tabular}

En la Fase de experiencia piloto y validación final del proyecto se espera la participación de una cuota de usuarios similar para cada perfil.

En este punto es necesario señalar la importancia de la gestión de aspectos éticos en el proyecto. Para cada una de las actividades, el supervisor explicó previamente las características del proyecto y de la prueba, y el participante debía firmar un consentimiento informado (ver ejemplo en Anexo).

\subsection{Actividades con participación de usuarios y resultados}

Diferentes métodos de investigación cuantitativa y cualitativa fueron empleados a lo largo del proyecto, seleccionando aquellas técnicas de participación más adecuadas para cada fase. Como norma, en todas ellas se utilizaron cuestionarios de evaluación de la experiencia de usuario construidos a partir de escalas tipo Likert, que permiten evaluar y comparar la satisfacción con la tecnología en una escala de o a roo puntos. Una descripción detallada de este proceso, las técnicas y materiales y los resultados de las evaluaciones, puede encontrarse en Madrid et al. (2013; 20I4). 


\section{Fase de recogida de requisitos}

En las primeras etapas del proyecto se realizaron diferentes actividades para la recogida de información sobre las necesidades y preferencias de las personas con discapacidad y otros usuarios respecto a los cajeros automáticos.

I. Encuesta online: La encuesta consistía en 29 preguntas referidas a cinco secciones: perfil de discapacidad, información sociodemográfica general, información sobre familiaridad con la tecnología, información sobre la experiencia con el uso de cajeros y, más en concreto, con los cajeros de 'la Caixa'.

2. Entrevistas en profundidad: Del conjunto de personas que participaron en la encuesta, un grupo de 36 personas fueron entrevistadas para obtener un conocimiento más profundo de la razón de sus respuestas. Las preguntas se centraban en la frecuencia de uso de los cajeros, las barreras que encontraban y sus necesidades y preferencias de uso.
3. Test de usuario: Un grupo más reducido $(23$ personas) participó en el test de usuario, realizando tareas comunes con los cajeros automáticos como sacar dinero, consultar movimientos de cuenta o imprimir los recibos.

Los resultados ofrecieron información muy valiosa sobre cómo personas con diferentes capacidades acceden a los cajeros automáticos, los problemas que encuentran, las barreras de accesibilidad potenciales y las estrategias que usan para superarlas.

La Figura I muestra resultados de la encuesta online referidos a la experiencia de usuario con los cajeros automáticos existentes, y su comparación con las expectativas de los usuarios sobre los futuros cajeros APSIS4all. Puede observarse que la puntuación media para todos los participantes (All users) para los cajeros existentes es de 57 puntos sobre ıoo, lo cual puede considerarse muy modesto. Además, un análisis por perfiles muestra peores resultados para determinados grupos de usuarios, como es el caso de las personas con

\section{Figura 1. Evaluación de cajeros actuales vs. expectativas}

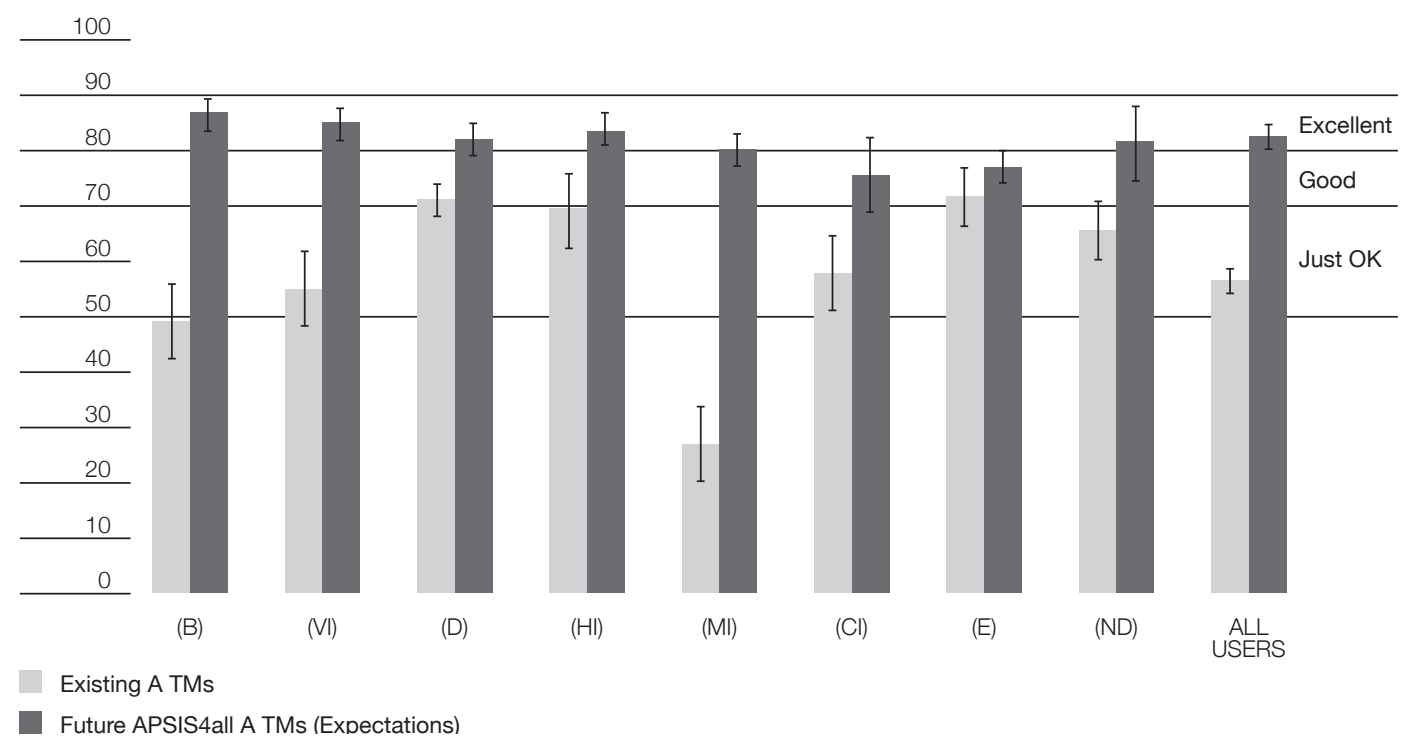

Fuente: Madrid et al. 20I4. 
discapacidad motriz $(M I)$ o las personas ciegas $(B)$ que no alcanzan los 50 puntos. Una vez explicadas las funcionalidades que aportarán los nuevos cajeros de APSIS 4 all, las expectativas de todos los grupos de usuarios sobre estos nuevos cajeros se incrementaron por encima de los 70 puntos, lo cual es un indicio de que la aproximación adoptada en el proyecto es adecuada.

En general, puede decirse que las personas con discapacidad se enfrentan a algunas dificultades cuando usan los cajeros automáticos, que se vuelven barreras severas para perfiles de usuario específicos (por ejemplo, más de la mitad de los participantes con baja visión declararon experimentar problemas con el contraste de la pantalla cuando usaban un cajero).

\section{Fase de diseño y desarrollo iterativo}

La técnica de investigación seleccionada en esta fase fueron los test de usuario, en los que junto a la observación de la ejecución de diferentes tareas se utilizaron diferentes cuestionarios de evaluación de experiencia de usuario.

Un primer prototipo de la herramienta de recogida de información sobre necesidades y preferencias de usuario se desarrolló de acuerdo con los requisitos recogidos en la fase anterior. A partir de ahí, tres iteraciones de diseño fueron mejorando los prototipos con la participación de usuarios. Así, los participantes de diferentes perfiles fueron testeando las aplicaciones, valorando la usabilidad y accesibilidad de las mismas y señalando barreras aun existentes para su eliminación.

Como resumen que ilustra la importancia de contar con personas con discapacidad en un proyecto de $\mathrm{I}+\mathrm{D}+\mathrm{i}$ pueden mostrarse los siguientes gráficos de resultados:

La Figura 2 muestra los resultados de la evaluación de la experiencia de usuario de los prototipos de interfaces de cajero personalizados en sus primeras etapas, utilizando para ello una serie de escalas de o a roo puntos. Puede verse como, si tenemos en cuenta los resultados de todas las personas (All users), la puntuación otorgada por los participantes supera los 80 puntos. Esto podría considerarse un resultado excelente según el baremo de la escala utilizada. Sin embargo, un análisis detallado de la valoración aportada por los diferentes perfiles muestra diferencias importantes entre grupos de usuarios (por ejemplo las personas sin

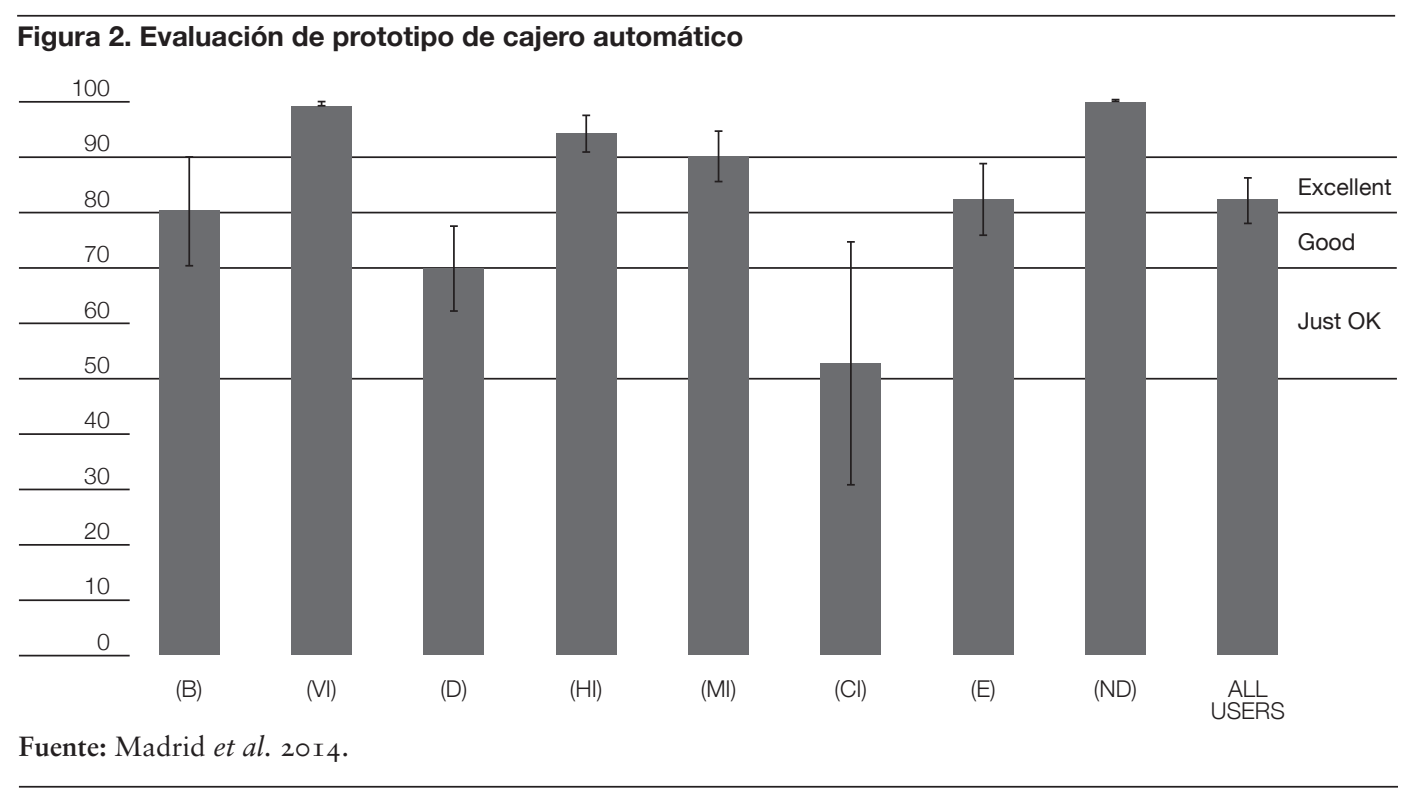




\section{Figura 3. Resultados en experiencia de usuario. Todos los participantes}
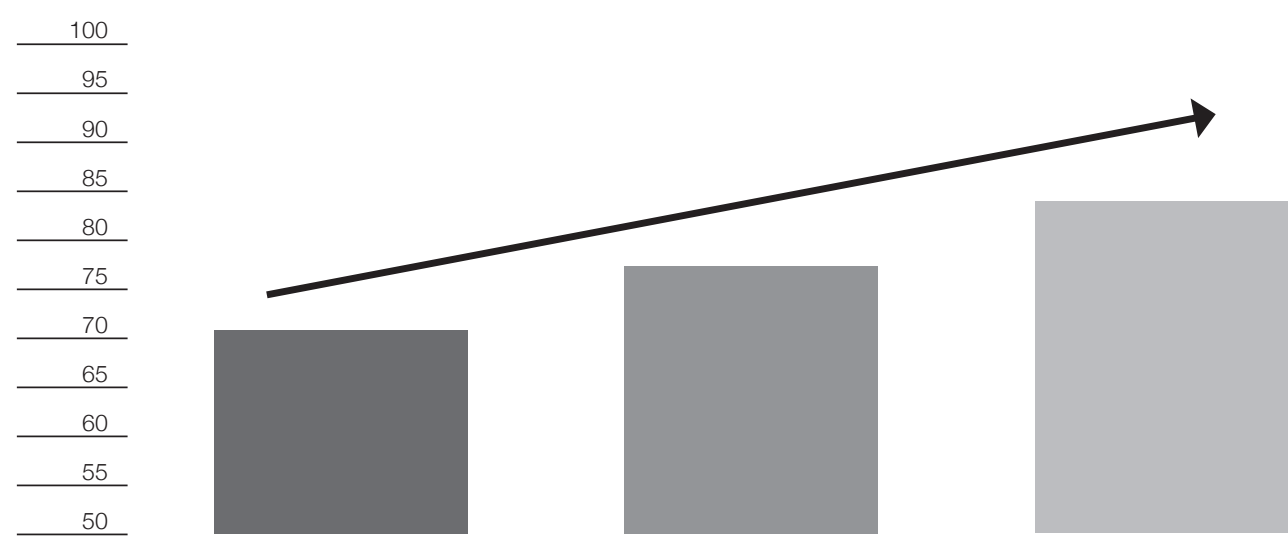

ITERACIÓN I
ITERACIÓN III discapacidad (ND) alcanzan los roo puntos, mientras que las personas con discapacidad cognitiva (CI) superan por poco los 50 puntos, que es una puntuación muy baja que denota barreras importantes). Esto confirma la importancia de tener en cuenta la perspectiva del diseño para todos, involucrando a diferentes perfiles de personas con discapacidad para conseguir que la tecnología sea usable para el mayor número de personas posible.

La Figura 3 muestra la mejora de la percepción de los participantes sobre la herramienta de recogida de necesidades y preferencias de usuarios a lo largo de sucesivas iteraciones. Lo primero a destacar es que ya los primeros prototipos tuvieron una aceptación considerable (70 puntos en la primera iteración). Este buen resultado difícilmente se hubiese producido sin la participación de personas con discapacidad en la Fase de recogida de requisitos. Por otra parte, cada evaluación realizada ha permitido ir refinando el sistema, a partir de las barreras encontradas y las sugerencias de los usuarios, de forma que en la iteración III la puntuación superaba los 80 puntos.

\section{Fase de experiencia piloto y validación}

El proyecto finaliza con una experiencia piloto, en la que las herramientas desarrolladas se despliegan para su uso normal en un contexto real, aunque controlado. En concreto, para este proyecto I+D+i 'la Caixa' ha desplegado Iooo cajeros automáticos con la solución APSIS 4 all en Madrid y Barcelona. Cualquier cliente de 'la Caixa' puede determinar sus necesidades y preferencias haciendo uso de la herramienta online $^{\mathrm{II}}$ y solicitar una nueva tarjeta en la que vendrán determinadas sus preferencias para el uso de los cajeros (por ejemplo uso de un lector de pantalla o de un interfaz simplificado). Con esta tarjeta, la persona podrá operar normalmente en estos cajeros.

En esta fase las personas con discapacidad participan de varias formas:

I. Encuesta de satisfacción y experiencia de usuario: Los usuarios que se registran en la herramienta online reciben una invitación para participar en una evaluación de los sistemas mediante cuestionarios. El objetivo del proyecto es que en esta experiencia piloto se registren 500 personas, y que entre un ro y un $20 \%$ pueda participar en la encuesta de satisfacción.

2. Monitorización de uso: Los datos de uso de las herramientas y tarjetas APSIS 4 all son monitorizados de forma anónima en el periodo del piloto. De esta forma, se

I I. http://cajerofacil.apsis4all.eus. 
podrán extraer datos para caracterizar este uso, compararlo con el uso de los cajeros estándar e idear mecanismos de mejora para el futuro. La explotación de estos datos permitirá además obtener información del total de la muestra y no solo de aquellos que aceptan participar en la encuesta de satisfacción, lo cual frecuentemente supone una limitación en este tipo de estudios.

3. Soporte y atención al cliente: Las personas con discapacidad participantes en el proyecto pueden contactar con el soporte del proyecto a través de correo electrónico. Las consultas recibidas permiten conocer nuevas necesidades y aclarar la información de cara a un futuro despliegue universal de la solución.

\subsection{Comunicación y difusión de resultados}

La comunicación y difusión de los resultados del proyecto de $\mathrm{I}+\mathrm{D}+\mathrm{i}$ es al mismo tiempo una obligación y una necesidad. Como se ha comentado antes, comunicar los resultados del proyecto a medida que se van produciendo ayuda a mantener la motivación y la involucración de los participantes con discapacidad. Por otra parte, es una responsabilidad ética el tener informados a los voluntarios de los resultados obtenidos en actividades en las que han participado.

En el caso de APSIS4all, algunas de las acciones realizadas para difundir los resultados del proyecto son:

I. A nivel científico-técnico, difusión del proyecto y sus resultados en diferentes congresos científicos y eventos (Bailey y Madrid, 20I4; Madrid et al., 20I3; Murtro, 20I3).

2. A nivel de organizaciones de personas con discapacidad, se realizan diferentes acciones como charlas informativas, envíos de correos electrónicos, contacto telefónico, etc.

3. A nivel de difusión a las personas con discapacidad, de forma más amplia, algunas de las acciones incluyen:
- Publicación de diferentes noticias en el portal Discapnet.

- Aparición del proyecto APSIS4all en el programa "Piensa en positivo" de RTVE $^{\text {I2 }}$.

- Contacto por email con participantes en fases anteriores del proyecto.

Todas estas acciones buscan no solo informar a los participantes individuales del proyecto $\mathrm{I}+\mathrm{D}+\mathrm{i}$, sino también difundir los avances del proyecto hacia la población general que puede ser beneficiada por el mismo, y hacia la industria que puede aprender de esta experiencia y aplicar los resultados a iniciativas similares.

\section{Conclusiones}

A lo largo de este artículo se han abordado las principales cuestiones prácticas para la involucración de personas con discapacidad en proyectos de $\mathrm{I}+\mathrm{D}+\mathrm{i}$ tecnológica. El enfoque que se describe, inspirado por nuevas aproximaciones como la investigación inclusiva o emancipadora, toma metodologías fundadas en el diseño para todos, el diseño centrado en el usuario y el diseño participativo, con el objetivo de que las personas con discapacidad tengan un papel más activo en la investigación y no solo como meros sujetos pasivos.

Las personas con discapacidad pueden y deben participar en todas las fases de un proyecto: ideación y planificación, recogida de requisitos, diseño y desarrollo de tecnología y experiencia piloto y validación. Sin embargo, el camino para permitir la participación de las personas con discapacidad en la investigación no está exento de dificultades, contando con barreras procedimentales y de comunicación que deben ser salvadas para que ningún colectivo quede excluido de los proyectos. A lo largo de este artículo se han repasado algunas estrategias

I2. ‘www.rtve.es/alacarta/videos/piensa-en-positivo/piensapositivo-30-I I-I $3 / 2$ I 84 I $48 /$ /. 
comunes basadas en nuestra experiencia en el área de Tecnologías accesibles de Technosite.

En concreto, y mostrando como ejemplo el caso del proyecto europeo APSIS $_{4}$ all, se ha ilustrado cómo puede reclutarse e involucrarse a personas con diferentes perfiles de discapacidad en un proyecto de $\mathrm{I}+\mathrm{D}+\mathrm{i}$ tecnológica. Los positivos resultados del proyecto sirven para ilustrar cómo la participación de personas con discapacidad permite el desarrollo tecnologías que, además de ser funcionales y accesibles, sean aceptadas y bien valoradas por sus futuros usuarios. Es importante sensibilizar a los investigadores y a aquellos responsables de desarrollos tecnológicos acerca de las ventajas de incluir a colectivos desfavorecidos en la participación de proyectos desde su conceptualización hasta la conclusión de los mismos, incluyendo en este proceso inclusivo y participativo las fases de experimentación y validación. 
Referencias bibliográficas

Alaminos, A. y Castejón, J. L. (2006): Elaboración, análisis e interpretación de encuestas, cuestionarios y escalas de opinión, Alicante: Universidad de Alicante.

Aldred, R. (2008): "Ethical and political issues in contemporary research relationships", Sociology, 42(5): 887-903.

Bailey, C. y Madrid, R.I. (2OI4): "Personalization services: Creating accessible public terminals", User Experience, I4(I) (en línea). <http:// uxpamagazine.org/personalization-services/>.

Barnes, C. (2003): "What a difference a decade makes: Reflections on Doing 'Emancipatory' disability research”, Disability \& Society, I $8(\mathrm{I}): 3-\mathrm{I} 7$.

Bühler, C. (200I): "Empowered participation of users with disabilities in universal design", Universal Access in the Information Society, I(2): 85-90.

De Benito, J. et al. (I ${ }^{\text {a }}$ Ed) (2010): Manual para un entorno accesible, Madrid: Real Patronato sobre Discapacidad.

Domenech, S. et al. (2OI3): "Involving older people in the design of an innovative technological system promoting active aging: the SAAPHO Project", Journal of Accessibility and Design for All JACCES, 3(I): I3-27.

España. Real Decreto Legislativo I/20I3, de 29 de noviembre, por el que se aprueba el Texto Refundido de la Ley General de derechos de las personas con discapacidad y de su inclusión social, Boletín Oficial del Estado, núm. 289, de 3 de diciembre de 2013, pp. 95635-95673.

España. Ley Orgánica I 5/I999, de I3 de diciembre, de Protección de Datos de Carácter Personal, Boletín Oficial del Estado, núm. 298, de I4 de diciembre de I999, pp. 43088- 43099.

Fundación ONCE (2009): Estudio experimental de las potencialidades del teléfono móvil como terminal capaz de interactuar con dispositivos dispensadores (cajeros automáticos) (en línea).
$<$ http://www.foal.es/sites/default/files/docs/37_ fonceinformeanexodifusion_o.pdf $>$.

FVE (2013): Acceso y uso de las TIC por las personas con discapacidad, Madrid: Fundación VODAFONE.

García Crespo, A. et al. (2012): "Servicios interactivos y accesibilidad en la Televisión Digital, una oportunidad para reducir la brecha digital”, Revista Iberoamericana de Tecnologías del/da Aprendizaje/Aprendizagem, 7(2): 86-93.

González Luna, B. (2013): "Investigando con personas con dificultades de aprendizaje", Revista Española de Discapacidad, I(2): 77-94.

Harris, J. y Roberts, K. (2003): “Challenging barriers to participation in qualitative research: Involving disabled refugees", International Journal of Qualitative Methods, 2(2): I 55-I66.

INE (20I 2): Encuesta de Integración Social y Salud (EISS 20I2), nota de prensa (en línea). <http:// www.ine.es/prensa/np8I7.pdf>.

Jiménez Lara, A. (20I I): El estado actual de la accesibilidad de las Tecnologías de la Información y la Comunicación (TIC), Madrid: CERMI/Fundación Vodafone.

Kitchin, R. (2000): “The researched opinions on research: disabled people and disability research", Disability \& Society, I 5(I): 25-47.

Madrid, R.I. et al. (20I4): "Improving the accessibility of Public Digital Terminals through personalisation: Comparison of direct and indirect interaction modes”. En: Stephanidis, C. \& Antona, M. (eds.): Universal Access in Human-Computer Interaction Design and Development Methods for Universal Access, Cham. SW: Springer.

Madrid, R.I. et al. (2013): “Applying HumanCentred Design to create a collecting tool of needs and preferences for the personalisation of ATMs”. En: Encarnação, P. et al. (eds.): Assistive Technology: From Research to Practice, Amsterdam: IOS Press. 
Muller, M.J. (2007): "Participatory design: The third space in HCI (revised)". En: Jacko, J. \& Sears, A. (eds.): Handbook of HCI 2 nd Edition, Mahway NJ USA: Erlbaum.

Murtro, J. (2013): Presentación de APSIS4all (Accessible Personalised Services In Public Digital Terminals for all) (video en línea). <http://www.discapnet.es/Castellano/ areastematicas/tecnologia/DRT4ALL/ES/ $\mathrm{DRT}_{4} \mathrm{ALLI}_{\text {I I }}$ /Paginas/programa 5.aspx $>$.

Naciones Unidas (2006): Convención sobre los derechos de las personas con discapacidad, Nueva York: Naciones Unidas (en línea). <http://www.un.org/esa/socdev/enable/ documents/tccconvs.pdf $>$.

Priestley, M. et al. (2010): “Towards a User-led agenda for disability research in Europe: learning from disabled people's organisations", Disability \& Society, $25(6)$ : 73 I-746.

Safayeni, F. et al. (2008): "Requirements engineering in new product development", Communications ACM, $5 \mathrm{I}(3)$ : 77-82.

Sainz de Salces, F. y Bustamante, J. (2OI4): "Aspectos éticos en la participación de personas con diversidad funcional en proyectos de investigación tecnológica", Revista Española de Discapacidad, 2(I): I65-I74.

Sánchez, J. (20I I): “En busca del Diseño Centrado en el Usuario (DCU): definiciones, técnicas y una propuesta", No Solo Usabilidad, Io (en línea). <http://www.nosolousabilidad.com/ articulos/dcu.htm>.

Stalker, K. (I998): "Some ethical and methodological issues in research with people with learning difficulties”, Disability \& Society, I3 (I): 5-I9.
Steinfeld, E. \& Meisel, J. (2OI 2): Universal design: Creating inclusive environments, Hoboken: John Wiley \& Sons, Inc.

Technosite (20I I): Monitoring eAccessibility in Europe: 20 I I Annual Report (en línea). $<$ http://www.eaccessibility-monitoring.eu/ researchResult.aspx $>$, acceso 30 de diciembre de 2013 .

Technosite (2008): Informe sobre la caracterización del colectivo de personas con necesidades especiales y su relación con el uso de las tecnologías, Madrid: Technosite.

Technosite (2007): La captación de muestras (y usuarios) en investigación cualitativa. Consideraciones técnicas, Madrid: Technosite.

Toboso-Martín, M. y Rogero-García, J. (2OI2): “ «Diseño para todos» en la investigacion social sobre personas con discapacidad", Revista Española de Investigaciones Sociológicas, I40: I63-I72.

Tullis,T. \& Albert, W (2008): Measuring the user experience: Collecting, analyzing, and presenting usability metrics, Burlington, MA: Morgan Kaufman.

Vines, J. et al. (2013): "Designing for- and withvulnerable people", Proceedings of $\mathrm{CHI}_{13}$, april 27-may 2, 20I3, Paris, France.

Walmsley J. \& Johnson, K. (2003): Inclusive research with people with learning disabilities: Past, present and futures, London: Jessica Kingsley.

Young, A. F. (2006): "Obtaining views on health care from people with learning disabilities and severe mental health problems", British Journal of Learning Disabilities, 34: I I-I9. 
Anexo. Proyecto APSIS4all. Hoja de consentimiento informado

Proyecto APSIS4all: Pruebas de usuario con herramienta web y cajeros automáticos

Gracias por tomar parte en este estudio.

- La persona coordinadora del proyecto me ha explicado el proyecto y estoy de acuerdo en participar en él.

- Entiendo que si tengo alguna pregunta de la Hoja de Información o de su explicación, puedo preguntar al investigador antes de que aceptar participar en el proyecto. Se me entregará una copia de esta Hoja de Consentimiento para guardarla.

- Entiendo que si decido, en cualquier momento de la investigación, que no deseo participar más en el proyecto, puedo hacerlo sin tener que dar explicación alguna. Más aún, entiendo que puedo retirar mis datos hasta tres semanas después de la fecha de la entrevista.

- Consiento el procesamiento de la información personal para las propuestas que me han explicado. Entiendo que tal información será tratada en concordancia con los términos de la Ley de Protección de Datos.

\section{Detalles de Contacto del participante:}

Nombre y Apellidos (en mayúsculas):

Género:

Edad:

Correo electrónico:

Teléfono:

Tipo de discapacidad (para participantes con discapacidad):

Detalles de Contacto del tutor legal (puede ser un testigo, cuidador, etc.): [lo siguiente será completado por el tutor legal del participante, si es aplicable]:

Nombre y Apellidos (en mayúsculas):

Relación con el participante:

Experiencia (si hay alguna; por ejemplo, cuidador, etc.):

\section{DECLARACIÓN DEL PARTICIPANTE:}

Yo estoy de acuerdo en que se me ha facilitado toda la información del proyecto de investigación y en qué consiste mi participación, y estoy de acuerdo en formar parte del estudio. He leído la Hoja de Información del proyecto arriba incluida y entiendo lo que el estudio de investigación implica.

Fecha:

Lugar: Technosite - C/ Albacete, $32^{\mathrm{a}}$ planta 28027 - Madrid.

Firma del participante: 


\section{DECLARACIÓN DE LOS TUTORES LEGALES/TESTIGOS [cuando proceda]:}

Confirmo que el participante es incapaz de firmar el formulario de consentimiento pero ha aceptado participar en el proyecto. Los organizadores han explicado las notas escritas arriba y la Hoja de Información sobre el proyecto y entiende lo que el estudio de investigación implica.

\section{Fecha:}

Lugar: Technosite - C/ Albacete, $32^{\mathrm{a}}$ planta 28027 - Madrid.

Firma del tutor/testigos:

Relación con el participante:

\section{DECLARACIÓN DEL INVESTIGADOR:}

Yo, confirmo que he facilitado la información al participante de la investigación sobre el estudio, lo que en mi opinión es adecuado y suficiente para que el participante entienda por completo la naturaleza, riesgos y beneficios del estudio, y los derechos del participante en la investigación. No ha existido coerción o influencia indebida. He sido testigo de la firma de este documento por parte del participante (y/o el tutor).

\section{Fecha:}

Lugar: Technosite - C/ Albacete, $32^{\mathrm{a}}$ planta 28027 - Madrid.

Firma del investigador: 


\section{HOJA DE CONSENTIMIENTO ADICIONAL PARA LOS CASOS DE PARTICIPANTES CON DISCAPACIDAD COGNITIVA O PERSONAS ILETRADAS (ADEMÁS DE LA QUE SE HA FIRMADO MÁS ARRIBA)}

Firmando más abajo acuerdo voluntariamente participar en este estudio de investigación. El estudio y la hoja de consentimiento me han sido explicados verbalmente en un idioma que comprendo. He tenido la oportunidad de preguntar todas mis dudas acerca de este estudio. He recibido respuesta a todas mis preguntas.

Fecha:

Lugar: Technosite - C/ Albacete, $32^{\mathrm{a}}$ planta 28027 - Madrid.

Nombre del participante (en letras mayúsculas):

Firma del participante:

Nombre del tutor (en letras mayúsculas):

Firma del tutor:

Nombre del investigador/supervisor (en letras mayúsculas):

Firma del investigador/supervisor: 